\title{
ALGUNOS ASPECTOS DE LA METATEORÍA DE PRINCIPIA IURIS*
}

\author{
Riccardo Guastini \\ Universidad de Génova
}

RESUMEN. El autor discute algunas tesis de la metateoría de L. FERRAJOLI. 1) En primer lugar, su concepción del objeto de la teoría del derecho, a saber, las estructuras formales de los sistemas jurídicos (el derecho se identifica simplemente con el conjunto de sus fuentes formales), lo que excluye del dominio de la investigación teórica el análisis lógico y pragmático de las doctrinas de los juristas, que son una parte importante del propio Derecho. 2) En segundo lugar, su concepción de las relaciones entre Derecho y lógica: en particular la idea según la cual los sistemas jurídicos deberían conformarse a los principios de la lógica deóntica (aunque de hecho no lo hagan), del tal forma que deberían ser completos y consistentes; así como la idea de que por un lado hay relaciones de implicación lógica entre normas, pero por otro lado las normas lógicamente implícitas no pertenecen al sistema jurídico si no han sido positivamente promulgadas. 3) En tercer lugar, su crítica de ciertas falacias que, en su opinión, serían típicas del normativismo y del realismo jurídico (esencialmente: la confusión sistemática entre Derecho válido y Derecho vigente). jurídico.

Palabras clave: FERRAJolı, sistema jurídico, lógica deóntica, normativismo, realismo

ABSTRACT. The author discusses a number of theses pertaining to L. FERRAJOLI's meta-theory. 1) First, his conception of the subject matter of legal theory, viz. the formal structures of legal systems (the law being simply identified with its formal sources), which casts outside from theoretical investigation the logical and pragmatic analysis of lawyers' doctrines and reasoning, which in fact are a significant part of the law itself. 2) Secondly, his conception of the relationships between law and logic -in particular, the idea according to which legal systems ought to comply with the principles of deontic logic (although actually they do not), and hence ought to be consistent and gapless; and the idea according to which relationships of logical entailment among norms do exist, but nevertheless no logically implicit norm belongs to the legal system until it is actually promulgated. 3) Thirdly, his criticism of certain fallacies which, in his view, are typical of both normative and realistic theories of law (most of all, the systematic confusion between valid law and the law in force).

Keywords: FERRAJOLI, legal system, deontic logic, normativism, legal realism.

* Fecha de recepción: 1 de diciembre de 2008. Fecha de aceptación: 18 de diciembre de 2008. 


\section{EL OBJETO DE LA TEORÍA DEL DERECHO}

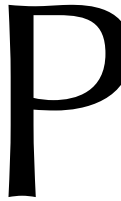

ara L. FERRAJOLI (en adelante, LF) ${ }^{1}$ — como para el BobBio de los años cincuenta $^{2}$ - el objeto de la teoría del Derecho es la identificación y el análisis «de las estructuras formales de los ordenamientos jurídicos independientemente de los valores que les dan forma y de sus variables y concretos contenidos prescriptivos» (p. 3); la teoría del derecho se dirige, entonces, «no al estudio de los contenidos normativos de un ordenamiento jurídico dado (que es la tarea típica de la dogmática), sino únicamente al análisis de las formas y de las estructuras del derecho positivo» (p. 4).

Sobre este punto, dos observaciones y un pequeño corolario.

i) Primera observación. La distinción entre teoría y metateoría es clara desde el punto de vista lógico: la metateoría es un metadiscurso del cual la teoría constituye el lenguaje-objeto. Pero la distinción no es igualmente tajante en el nivel de los hechos, por así decirlo: no solamente en el sentido (obvio) en el que en un mismo discurso se pueden mezclar distintos niveles de lenguaje, sino también en el sentido en que en ocasiones una tesis teórica tiene implicaciones (en sentido amplio) metateóricas y viceversa. Veamos a continuación un ejemplo.

La idea de que la teoría del Derecho tiene por objeto las «estructuras formales» de los ordenamientos (que es una tesis metateórica) presuponía en BOBBIO y presupone en LF una concepción peculiar del Derecho (es decir, una tesis teórica): aquella concepción que identifica el Derecho con el conjunto de los documentos normativos (con el «lenguaje del legislador», como decía BOBBIO 3 , y como repite LF, p. 9). Tal concepción del Derecho ${ }^{4}$ es problemática y, desde una mirada desencantada, parece algo ingenua.

La misma supone que las operaciones intelectuales llevadas a cabo por los juristas - la interpretación, las construcciones dogmáticas, la explicitación de normas no expresas (que se pretenden implícitas), etc. - sean un quid externo al Derecho y no una parte integrante del mismo. Supone que se puede hablar sensatamente del derecho (de los textos normativos) independientemente de las elaboraciones conceptuales, de las decisiones interpretativas, y de las operaciones de construcción jurídica llevadas a cabo por la dogmática 5 .

A esta concepción se le puede señalar que los textos normativos no tienen, por así decirlo, vida propia independientemente de la interpretación y de la dogmática, y que por tanto aquello que llamamos «el Derecho» es indistinguible de los conceptos y de las doctrinas que usan los juristas, aparentemente para describirlo en el nivel de metalenguaje, en realidad para modelarlo. Desde este punto de vista, en resumen, el Dere-

${ }^{1}$ L. FerRAJOlI, 2007: Principia Juris. Teoria del diritto e della democrazia, vol. I, Teoria del diritto, RomaBari. Las referencias a esta obra son señaladas entre paréntesis directamente en el texto.

${ }^{2}$ N. BobBIO, 1955: Studi per una teoria generale del diritto, Torino.

${ }^{3}$ N. Boвbio, 1950: «Scienza del diritto e analisi del linguaggio», ahora bajo el título «Scienza giuridica» en N. BobBio, Contributi ad un dizionario giuridico, Torino.

${ }^{4}$ Que se remonta, al menos, a J. Austin, 1832: The Province of Jurisprudence Determined.

5 Esta concepción supone también que el Derecho se agote en los textos normativos y que no incluya — como, por el contrario, quería KELSEN— las «normas individuales» creadas por las decisiones de los tribunales, de la administración, y por la autonomía privada. 
cho es —en todo tiempo y lugar - simplemente indistinguible, como habría dicho G. TARELLO, de la «cultura jurídica» ${ }^{6}$.

Esta concepción alternativa (aquí apenas esbozada, evidentemente) sugiere para la teoría del derecho un objeto un tanto diverso del que le parece apropiado a LF: no las estructuras formales del ordenamiento, entendido como discurso del «legislador» (en el sentido genérico de autoridad normativa), sino el análisis lógico y pragmático de los discursos de la «jurisprudencia», entendida obviamente como sapientia juris.

Así entendida, la teoría del Derecho se reduce — para utilizar una expresión puesta en circulación por BOBBIO- a «metajurisprudencia» ${ }^{7}$ o, si se prefiere, a filosofía de la ciencia jurídica. Como parte integrante de la misma se encuentra, naturalmente, la teoría de la interpretación y de la argumentación, ausente, quizás no por casualidad, en el libro de LF.

En la concepción de LF teoría del Derecho y «ciencia jurídica» (en el sentido de dogmática o doctrina) no tienen relaciones lógicas evidentes. En la concepción alternativa, la teoría del Derecho es un metalenguaje del cual la «ciencia jurídica» constituye el lenguaje objeto.

ii) Segunda observación. Qué cosa sean las «estructuras formales» del derecho positivo no está del todo claro (no lo ha estado nunca, ni siquiera cuando BOBBIO se refería a ellas). Se comprende, en negativo, que la teoría del derecho prescinde del contenido prescriptivo de uno u otro ordenamiento. Pero, en positivo, ¿cuál es exactamente su objeto? En otra ocasión he arriesgado la hipótesis de que las estructuras formales del Derecho se identifican: por un lado, con el «néustico» de las normas; por otro lado, con el sistema de las fuentes ${ }^{8}$. Si es así, parece entonces que la teoría del Derecho se resume en una combinación de lógica deóntica y de teoría de las fuentes.

Sin embargo la lógica deóntica, como también se preocupa por subrayar LF en muchas ocasiones, vale (si vale) para cualquier conjunto de normas, y no sólo para las normas jurídicas. Y la teoría de las fuentes, por otra parte, es difícilmente distinguible de (una parte de) la ciencia del Derecho constitucional. En un cierto sentido la lógica deóntica es demasiado «formal» para la teoría del derecho y la Teoría de las fuentes no lo es suficientemente (no lo es completamente).

También se podría agregar esto: los conceptos teóricos serían estrictamente formales —en el sentido de indiferentes al contenido prescriptivo de los distintos ordenamientos- si, y sólo si, fuesen idóneos para describir cualquier ordenamiento positivo posible (sin coordenadas espacio-temporales). Pero es lícito dudar de que conceptos de este tipo se puedan construir. Sería posible construirlos si, y sólo si, todos los ordenamientos presentasen algunas características comunes. Y eso no es en absoluto cierto. Parece más sabio considerar los conceptos de la teoría general como idóneos para describir no cualquier ordenamiento (presente, pasado, o futuro), sino sólo grupos homogéneos de ordenamientos, similares y comparables: concretamente, los ordenamientos occidentales modernos.

\footnotetext{
${ }^{6}$ Cfr. por ej. G. Tarello, 1974: Diritto, enunciati, usi, Bologna, 1974, pp. 329 ss.

7 N. BobBIO, 1967: «Essere e dover essere nella scienza giuridica», actualmente en N. BobBIO, 1970, Studi sulla teoria generale del diritto, Torino.

8 R. Guastini, 1996, Distinguendo, Torino, 45.
} 
El corolario de estas observaciones es el siguiente. Para LF la teoría del Derecho es un discurso (casi) completamente artificial: un conjunto de estipulaciones. Para quien concibe la teoría del Derecho como metajurisprudencia — como análisis de una cultura jurídica espacio-temporalmente determinada - las cosas son diferentes.

Por una parte, la tarea de la teoría no es tanto construir conceptos nuevos, sino más bien reconstruir los conceptos empleados por los juristas: por tanto, no estipular, en el sentido más estricto, sino más bien redefinir. Por otra parte, la tarea de la teoría no es sólo el análisis de los conceptos (la investigación sobre el significado de las palabras), sino también el análisis lógico de los razonamientos y el análisis pragmático de las doctrinas de los juristas.

\section{LÓGICA Y DERECHO}

Según LF, la lógica, en particular la lógica deóntica, es un conjunto de prescripciones, un conjunto de «principios [...] normativos» (p. 28) que, aun siendo «externos» al derecho, todavía «imponen al derecho positivo [...] la lógica que éste de hecho no posee, pero que de derecho debe tener» (p. 28, la cursiva es mía). La lógica, en fin, gobierna el Derecho: no en el sentido que el Derecho sea conforme a la lógica, sino en el sentido que debería serlo.

Esto quiere decir, si no entiendo mal, que la lógica es vinculante para el legislador como si estuviese incorporada a la constitución (a una constitución rígida, se entiende) al modo de un «derecho sobre el derecho», como las normas sobre la producción jurídica?.

Ahora bien, que la constitución (una constitución cualquiera) incorpore los principios de la lógica es algo, obviamente, implausible ${ }^{10}$. Sin embargo, es perfectamente plausible que la lógica deóntica sea un modelo de legislación racional, como ya lo ha sugerido VON WRIGHT ${ }^{11}$. Esta idea merece algunos comentarios.

Según LF, los principios que la lógica deóntica impone al legislador serían dos: el principio de coherencia y el principio de plenitud o completitud ${ }^{12}$.

i) El principio de coherencia - una conducta no puede ser permitida y no permitida - prohíbe al legislador introducir antinomias en el sistema jurídico, en particular antinomias entre dos normas de distinto rango en la jerarquía de las fuentes. Así,

\footnotetext{
9 La lógica deóntica, en otras palabras, tiene en la teoría de LF un papel análogo a lo que tiene el derecho natural en algunas versiones del iusnaturalismo.

${ }^{10}$ En realidad yo creo que el derecho positivo no sólo no incorpora la lógica, sino que por el contrario: a) puede autorizar inferencias lógicamente inválidas (por ej., inferencias por analogía), y b) puede impedir inferencias que serían lógicamente válidas (una norma implícita tácitamente derogada ya no puede ser inferida aunque sean todavía válidas las normas que la implican). Cfr. R. GUASTINI, 2001, «Cinco observaciones sobre validez y derogación», en Discusiones, n. 2, pp. 62 y ss.

${ }^{11}$ G. H. vON Wright, 1982: «Norms, Truth, and Logic» (1982), en G. H. vON Wright, 1983: Practical Reason, Oxford.

${ }_{12}$ Obsérvese que ambos principios gobiernan no un tipo de razonamiento, sino la propia formación del lenguaje legislativo. Personalmente, comparto la tesis de LF de que la lógica es una disciplina prescriptiva. Creo, sin embargo, que las prescripciones de la lógica sirven para controlar la validez de los razonamientos, no la formación de las premisas.
} 
por ejemplo, el legislador (ordinario) no puede prohibir aquello que una norma constitucional permite.

ii) El principio de completitud tiene dos implicaciones distintas (aunque LF desarrolle sólo la segunda, de modo tal que me pregunto si la primera no es, tal vez, preterintencional).

ii.i) En primer lugar, una conducta no puede ser deónticamente incalificada, puesto que tal principio ordena al legislador calificar deónticamente cada conducta posible.

Esto deriva claramente del postulado según el cual «todo comportamiento supone la existencia de una modalidad deóntica de la cual es argumento» (p. 91). Lo que equivale a decir, me parece, que cualquier comportamiento sólo puede ser o permitido o no permitido: no se dan comportamientos deónticamente indiferentes. Se sigue que todo sistema deóntico es necesariamente completo, herméticamente cerrado, en el sentido que no existe conducta que no sea deónticamente calificada dentro del sistema.

ii.ii) En segundo lugar, se dan relaciones de implicación lógica entre normas, pues el principio ordena al legislador (ordinario) deducir todas las implicaciones lógicas de las normas, en particular de las normas superiores (constitucionales): si por ejemplo una norma constitucional (perteneciente a una constitución rígida) confiere un derecho subjetivo, el legislador debe establecer la obligación correspondiente, ya que toda norma que confiera un derecho implica lógicamente una norma que impone una obligación.

La idea de que el principio de coherencia es, en algún sentido, «vinculante» para un legislador racional no es nueva — se encuentra en VON WRIGHT y también en Ross ${ }^{13}$ y da forma a una intuición difundida. Además, se puede sostener que el principio de coherencia está realmente incorporado a muchas constituciones vigentes bajo la forma de principio de igualdad.

En referencia a los dos aspectos del principio de completitud, pueden realizarse dos observaciones, sobre todo con el objetivo de subrayar la originalidad de la posición de LF.

Primera observación. Sobre la tesis de que cualquier comportamiento sólo pueda ser o permitido o no permitido - puesto que no se dan comportamientos deónticamente indiferentes - se puede estar de acuerdo, a la condición, por supuesto, de entender «permiso» en sentido débil, o sea en el sentido de no prohibido ${ }^{14}$.

A primera vista, la tesis de LF podría ser entendida simplemente como una nueva formulación de la doctrina de la completitud necesaria de todo sistema normativo, argumentada a través del «principio de prohibición» (la norma general negativa de ZITELMANN ${ }^{15}$,

13 A. Ross, 1968: Directives and Norms, London, cap. VI.

${ }^{14}$ Es un tanto sorprendente que en el libro de LF, al menos prima facie, no existan trazos de la distinción metateórica fundamental entre normas y proposiciones normativas ( $c f r$. por ej. E. BULYGIN, 1982: «Norms, Normative Propositions, and Legal Statements», in G. Floistad (ed.), Contemporary Philosophy. A New Survey, vol. 3, The Hague, 1982), de la cual se sigue la distinción ulterior entre «permitido» en sentido débil, i.e. no prohibido, $\mathrm{y}$ «permitido» en sentido fuerte, i.e. positivamente calificado como permitido por una norma (G. H. VON Wright, 1963: Norm and Action, Oxford; C. E. AlChOuRRÓn y E. BulYgIn, 1971: Normative Systems, Wien).

${ }^{15}$ E. Zitelmann, 1903: Lücken im Recht, Leipzig. 
la norma general excluyente de DONATI ${ }^{16}$ ), ya definitivamente criticado por ALCHOURRÓN y BULYGIN ${ }^{17}$.

Pero no se debe olvidar que el principio de completitud —como los principios lógicos en general- son, para LF, principios directivos de legislación racional: no pretenden describir ningún sistema normativo existente, sobre todo no un sistema dinámico (como lo es el derecho positivo), pero pretenden dirigir a - para usar la fórmula de Bobbio- «el buen legislador» ${ }^{18}$. De modo tal que el principio de completitud parece resumirse en esto: en la recomendación al legislador (¿o al constituyente?) de formular expresamente la norma general excluyente: «Todo aquello que no está expresamente prohibido está permitido», para cerrar el sistema jurídico ${ }^{19}$.

Sugerencia, esta, bastante original, ya que, si no me equivoco, todos aquellos que hasta ahora han elucubrado sobre la completitud necesaria del Derecho siempre han sostenido que la norma general excluyente ya pertenece al Derecho (a cualquier sistema jurídico), aunque no esté expresamente formulada.

Segunda observación. LF, si entiendo bien, sostiene dos ideas notables: por un lado, la idea de que se dan relaciones de implicación lógica entre normas; por otro, la idea de que sin embrago las normas implícitas no pertenecen al sistema jurídico hasta que no sean expresamente formuladas por una autoridad normativa.

La originalidad de esta posición — que recuerda la distinción de CONTE entre validez deóntica y validez dianoética ${ }^{20}$ — llama la atención si se confronta con la del último KELSEN $^{21}$. Por una parte, LF no comparte la tesis del último KeLSEN, según la cual no se dan relaciones lógicas entre normas. Por otra, LF lleva, sin embargo, a consecuencias extremas la tesis, también del último KELSEN, según la cual en el Derecho no existen normas que no sean «puestas», expresamente formuladas por alguna autoridad normativa.

Sobre este punto me limito a señalar tres problemas.

Uno. Las dos ideas de LF parecen discordantes entre sí. Si se dan relaciones lógicas - en particular, de implicación lógica- entre normas, entonces una norma lógicamente implícita debería existir en el sistema normativo del que se habla sin que se requiera formularla y promulgarla. Si, por el contrario, ninguna norma «existe» hasta que no sea formulada y promulgada, entonces no se dan relaciones de implicación lógica entre normas, o tales relaciones son jurídicamente irrelevantes. Para armonizar estas ideas - hijas directas de la tesis de que la lógica sea vinculante para el legislador pero no incorporada al derecho positivo- se requeriría quizás un complemento de argumentación.

Dos. La tesis según la cual se dan relaciones lógicas entre normas es notoriamente problemática (y la cuestión hasta ahora no está resuelta): por eso, la tesis requeriría

${ }^{16}$ D. Donati, 1910: Il problema delle lacune dell'ordinamento giuridico, Milano, (ahora en D. DonATI, 1966: Scritti di diritto pubblico, I, Padova).

${ }_{17}$ C. E. Alchourrón y E. Bulygin, Normative Systems, cit., pp. 125 y ss.

${ }_{18}$ N. BoвBIO, 171: «Le bon législateur», en Logique et analyse, n. 53-54.

19 «Tout ce qui n'est pas défendu par la loi ne peut être empêché, et nul ne peut être contraint à faire ce qu'elle n'ordonne pas» (Déclaration des droits de l'bomme et du citoyen, art. 5).

20 A. G. CONTE, 1986: «Deontico vs. dianoetico», en Materiali per una storia della cultura giuridica, n. 2. 
alguna fundamentación filosófica (existen diversas a disposición ${ }^{22}$, que van desde la lógica de la satisfacción hasta la lógica sin verdad).

Tres. La tesis según la cual las normas implícitas (incluso aquellas implícitas en sentido estricto, es decir en sentido lógico) no pertenecen al sistema jurídico —o sea la tesis según la cual el sistema jurídico no incluye todas las consecuencias lógicas de las normas puestas - contradice no sólo una conocida teoría de los sistemas normativos (aludo obviamente a ALCHOURRÓN y BULYGIN ${ }^{23}$ ), sino también la intuición común de los juristas ${ }^{24}$. Aquí también un complemento de argumentación sería bienvenido.

Finalmente, una nota marginal para concluir este punto. Las dos implicaciones (o aplicaciones) del principio de completitud suponen dos conceptos distintos de laguna. Bajo la la primera implicación, debe entenderse por laguna la omisión de calificación deóntica de un comportamiento (que es el concepto «clásico» de laguna normativa $^{25}$, puesto en discusión por ALCHOURRÓN y BULYGIN). Bajo la segunda, por laguna debe entenderse la omisión de promulgación de una norma que sería condición necesaria (no suficiente) para la efectividad de otra norma, o sea lo que se suele llamar «laguna técnica».

\section{LA CRÍTICA DE LAS FALACIAS}

Uno de los aspectos más notables de la metateoría de LF es el proyecto de crítica de cuatro falacias ideológicas:

«a) la falacia iusnaturalista, que confunde la validez de las normas con su justicia, impidiendo reconocer la existencia de normas válidas aunque injustas; b) la falacia ético-legalista, que confunde la justicia con la validez, impidiendo reconocer la existencia de normas injustas aunque válidas; c) la falacia normativista, que confunde el vigor de las normas con su validez, impidiendo reconocer la existencia de normas vigentes aunque inválidas; d) la falacia realista, que confunde la validez con la vigencia o incluso con la eficacia, impidiendo reconocer la existencia de normas válidas o de todos modos vigentes aunque ineficaces, o. peor, su invalidez o incluso su inexistencia sólo porque son eficaces» (p. 42).

Sobre esto no se puede no estar de acuerdo. Quiero solamente observar, para evitar equívocos, que sería no obstante un error reducir el normativismo y el realismo a las respectivas falacias en las cuales tanto uno como el otro pueden incurrir contingentemente. Tanto «normativismo» como «realismo» son términos algo equívocos (por ejemplo son usados en algunas ocasiones para designar una familia de teorías del derecho y en otras para designar una familia de teorías de la ciencia jurídica) y de todos modos vagos (porque justamente se refieren no a teorías, sino a familias de teorías). De todos

${ }^{21}$ H. KelSEn, 1965: «Diritto e logica», trad. it. en P. COMANDuCCI y R. GuASTINI (eds.), L'analisi del ragionamento giuridico. Materiali ad uso degli studenti, vol. II, Torino.

${ }^{22}$ Cfr., p. ej., E. BulYGin, 1995: «Lógica deóntica», en C. E. Alchourrón (ed.), Lógica, Enciclopedia Iberoamericana de Filosofía, vol. VII, Madrid.

${ }^{23}$ C. E. AlChOURRón y E. BulYgin, Normative Systems, cit.

${ }^{24} \mathrm{O}$, mejor dicho, varias intuiciones de los juristas, inclusa la intuición según la cual la norma individual contenida en el dispositivo de un fallo está justificada si, y sólo si, ha sido deducida de una norma general (junto con una proposición que describe los hechos del caso).

${ }_{25}$ Sobre esto versa el libro de A. G. CONTE, 1962: Saggio sulla completezza degli ordinamenti giuridici, Torino. 
modos, creo que se puede convenir que poseen un núcleo de significado generalmente compartido que, grosso modo, sería el siguiente.

Por normativismo se entiende comúnmente el punto de vista según el cual el Derecho es un conjunto de normas válidas (según los criterios de validez del sistema jurídico del que se trata), y la tarea de la ciencia jurídica es describir (el contenido de las) normas y no otra cosa: no lo que «es», sino lo que «debe ser».

Por realismo se entiende comúnmente el punto de vista según el cual el Derecho es el conjunto de las normas efectivamente usadas por los órganos de aplicación en la justificación de sus decisiones (normas por tanto vigentes, pero vigentes en un sentido ligeramente diferente de aquel que LF atribuye a esta palabra).

Ahora bien, si existe un acuerdo sobre estas definiciones mínimas, se puede decir lo siguiente:

i) El normativismo, en cuanto tal, no se resuelve necesariamente en una ideología falaz. Más bien yo diría que tiende a resolverse en un programa de ciencia jurídica que parece justamente coincidir con el que propugna LF.

Una descripción del Derecho de inspiración normativista, fiel a la idea de que es tarea de la ciencia jurídica describir lo que «debe ser», debería consistir precisamente en describir, entre otras cosas, lo que «debe ser» según la constitución, y por tanto en constatar aquellos fenómenos de invalidez (material) de la legislación ordinaria que son tan caros a LF. La ciencia jurídica de inspiración normativista es indistinguible de (una parte de) la dogmática.

ii) También el realismo, en cuanto tal, no se resuelve necesariamente en una ideología falaz. Se resuelve más bien en una descripción adiáfora — desde un punto de vista externo- de las normas efectivamente aplicadas por los órganos de aplicación (que no son sólo los tribunales): con independencia, ciertamente, de la validez de las propias normas, ya que de hecho sucede que los órganos de aplicación aplican normas inválidas y no dan aplicación a normas válidas.

Una descripción realista del Derecho vigente es una empresa científica puramente empírica, que no contribuye en ningún modo a la dogmática —no dice nada acerca de lo que «debe ser»- ni pretende sustituirla. Pero, ¿existe quizás otro modo, no empírico, de conocer el Derecho vigente? Siempre que no se quiera sostener que el conocimiento empírico del derecho carece de utilidad alguna... ${ }^{26}$.

(Traducción de Erica Frontini)

${ }^{26}$ En un cierto sentido, es lo que sostenía KeLSEN con el argumento de que la jurisprudencia normativa describe en términos de «deber ser» precisamente esas mismas normas que la jurisprudencia realista pretende describir en términos de probabilidad de aplicación, porque la jurisprudencia normativa describe normas válidas dentro de un ordenamiento en su conjunto eficaz, y por tanto normas que probablemente serán obedecidas o aplicadas. H. KeLSEN, 1945: General Theory of Law and State, Cambrige (Mass.), 169 ss. 